\title{
On the estimate of magnetic non-potentiality of sunspots derived using Hinode SOT/SP observations: Effect of polarimetric noise
}

\author{
Sanjay Gosain, Sanjiv Kumar Tiwari and P. Venkatakrishnan \\ Udaipur Solar Observatory, Physical Research Laboratory, Udaipur, Rajasthan- 313 001, \\ INDIA
}

Received — 


\begin{abstract}
The accuracy of Milne-Eddington (ME) inversions, used to retrieve the magnetic field vector, depends upon the signal-to-noise ratio (SNR) of the spectropolarimetric observations. The SNR in real observations varies from pixel to pixel, therefore the accuracy of the field vector also varies over the map. The aim of this work is to study the effect of polarimetric noise on the inference of magnetic field vector and the magnetic non-potentiality of a real sunspot. To this end, we use Hinode SOT/SP vector magnetogram of a real sunspot NOAA 10933 as an input to generate synthetic Stokes profiles under ME model assumptions. We then add normally-distributed polarimetric noise of the level $0.5 \%$ of continuum intensity to these synthetic profiles and invert them again using $\mathrm{ME}$ code. This process is repeated 100 times with different realizations of noise. It is found that within most of the sunspot area ( $>90 \%$ area) the spread in the (i) field strength is less than 8 Gauss, (ii) field inclination is less than 1 degree, and (iii) field azimuth is less than 5 degrees. Further, we determine the uncertainty in the magnetic non-potentiality of a sunspot as determined by the force-free parameter $\alpha_{g}$ and Spatially Averaged Signed Shear Angle (SASSA). It is found that for the sunspot studied here these parameters are $\alpha_{g}=-3.5 \pm 0.37\left(\times 10^{-9} m^{-1}\right)$ and SASSA $=-1.68 \pm 0.014^{\circ}$. This suggests that the SASSA is a less dispersion non-potentiality parameter as compared to $\alpha_{g}$. Further, we examine the effect of increasing noise levels viz. $0.01,0.1,0.5$ and $1 \%$ of continuum intensity and find that SASSA is less vulnerable to noise as compared to $\alpha_{g}$ parameter.
\end{abstract}

Subject headings: Sunspot, Polarimetric noise, Magnetic field 


\section{Introduction}

Accurate determination of the vector magnetic field is very important for the studies of magnetic non-potentiality in active regions. The evolution of the active region magnetic field towards an increasingly non-potential state leads to buildup of magnetic free energy, i.e., energy above magnetic potential energy. This free magnetic energy is believed to drive the eruptive phenomena like flares and Coronal Mass Ejections (CMEs). Prediction of such phenomena on the Sun is very important for space weather forecasting, and requires good knowledge of the non-potentiality of the magnetic field in solar active regions.

Force-free parameter $\alpha_{g}$ has been studied to infer the non-potentiality of sunspots for a long time (Pevtsov et al. 1994, 1995; Abramenko et al. 1996; Bao \& Zhang 1998; Hagino \& Sakurai 2004, 2005; Nandv 2006). Using the second moment of minimization (Hagino \& Sakurai 2004; Tiwari et al. 2009a) the relation between $\alpha_{g}$ and the vector field components is given by the following relation :

$$
\alpha_{g}=\frac{\sum\left(\frac{\partial B_{y}}{\partial x}-\frac{\partial B_{x}}{\partial y}\right) B_{z}}{\sum B_{z}^{2}}
$$

The global alpha $\left(\alpha_{g}\right)$ actually gives twice the degree of twist per unit axial length and not the twist (see Appendix A of Tiwari et al. (2009a)). Thus, if the length of the magnetic field structure in a volume is given, global twist can be obtained from alpha for force-free fields.

Another parameter, spatially averaged signed shear angle (SASSA), henceforth

denoted as $\widehat{\Psi}$, was recently proposed by Tiwari et al. (2009b) as a measure of magnetic non-potentiality in sunspots. This parameter is the spatial average of the angle between observed magnetic field and potential field azimuth. It is derived from the following relation.

$$
S A S S A \quad \text { or } \quad \widehat{\Psi}=\left\langle\operatorname{atan}\left(\frac{B_{y o} B_{x p}-B_{y p} B_{x o}}{B_{x o} B_{x p}+B_{y o} B_{y p}}\right)\right\rangle
$$

where $B_{x o}, B_{y o}$ and $B_{x p}, B_{y p}$ are observed and potential transverse components of the 
magnetic field, respectively. The angled-braces represent the spatial average taken over all pixels except those below noise (see section 2.3). This parameter thus gives average shear angle on the photospheric boundary and is independent of the force-free nature as well as shape of the sunspots (Venkatakrishnan \& Tiwari 2009). The high-quality Hinode data has also allowed these authors to study the contribution of local alpha values of umbral and penumbral structures to global alpha value of the sunspots. Further, Gosain et al. (2009) evaluated $\widehat{\Psi}$ of active region NOAA 10930 during X-class flare of 13 December 2006 using vector magnetograms obtained few hours before and after the flare by Spectro-Polarimeter (SP) instrument (Lites et al. 2007; Ichimoto et al. 2008) with Solar Optical Telescope (SOT) onboard Hinode satellite (Kosugi et al. 2007; Tsuneta et al. 2008), and found that the $\widehat{\Psi}$ decreased after the flare. However, high-cadence vector magnetograms are needed to follow the evolution of non-potentiality, characterized by $\widehat{\Psi}$, during the flare interval. Magnetographs based on tunable filters like Imaging Vector Magnetograph (IVM) (Mickey et al. 1996) and Solar Vector Magnetograph (SVM) (Gosain et al. 2004, 2006) from ground and recently flown Helioseismic and Magnetic Imager (HMI) onboard Solar Dynamics Observatory (SDO) from space (Scherrer \& SDO/HMI Team 2002) can provide such high-cadence magnetograms of active regions.

Tiwari et al. (2009a) had earlier evaluated the effect of polarimetric noise on the magnetic twist parameter $\alpha_{g}$ and magnetic energy using a synthetic bipole (Low 1982). However, for real sunspots the distribution of magnetic field vector is not the same as in the case of synthetic bipole. Real sunspots have umbra and penumbra besides fine structure, as seen by high resolution ground based and space based Hinode observations. In a recent study by Su et al. (2009) using Hinode observations it was found that the current and $\alpha$ distribution in a sunspot is not smooth but has a fine mesh-like structure with mixed polarity patches of $\alpha$ in the umbra and radial spine like pattern with alternating polarity in penumbra of a sunspot. At the umbra penumbra boundary they found an incomplete 
annular ring with current and $\alpha$ values of sign opposite to that of global $\alpha$ i.e., $\alpha_{g}$. Further, Tiwari et al. (2009b) have shown that the distribution of $\alpha$ in penumbra almost cancels to zero while in umbra there is a net value which bears same sign as $\alpha_{g}$ and has same magnitude as $\alpha_{g}$. Further, the new parameter $\widehat{\Psi}$ that has been introduced recently by Tiwari et al. (2009b) needs to be assessed for robustness in the presence of uncertainties in the magnetic field parameters of a real sunspot. This work therefore extends the results of Tiwari et al. (2009a) to the case of a real sunspot observed by Hinode and to the new parameter $\widehat{\Psi}$.

The uncertainties in vector magnetic field obtained by fitting the observed Stokes profiles with model profiles can arise mainly due to the following reasons : (i) invalidity of the model atmosphere used, e.g., ME model cannot fit asymmetric Stokes profiles which arise due to variation of physical parameters within the line forming region, and (ii) polarimetric noise, e.g., sensitivity in determining the field parameters depends on the Signal to Noise Ratio (SNR) of the Stokes profiles. The former reason has been evaluated by Westendorp Plaza et al. (2001) by comparing ME and SIR (Stokes Inversion based on Response-Functions; Ruiz Cobo \& del Toro Iniesta (1992)) inversions. SIR inversions yield variation of physical parameters within the line forming region, by exploiting the asymmetric nature of Stokes profiles. They have shown that using ME inverted field parameters is equivalent to using field parameters averaged over the line forming region obtained by SIR inversions. So, neglecting effect of Stokes asymmetry we focused on the errors in the field measurements due to polarimetric noise.

The paper is organized as follows. In section 2 we describe the Hinode SOT/SP observations and the method of simulating noisy Stokes profiles and their inversion. The results are presented in section 3. The importance of the non-potentiality parameters and the inaccuracy in their determination as a result of polarimetric noise is discussed in 
section 4 . Section 5 gives the conclusions based on the present study and the future studies required.

\section{Data and Method}

\subsection{Observational Data}

The high resolution spectro-polarimetric observations were made by the SpectroPolarimeter (SP) instrument working with Solar Optical Telescope (SOT) onboard Hinode space mission. We choose a regular isolated sunspot in NOAA 10933 during 05 January 2007, when it was located close to the disk center $(\mu=0.99)$ during 07:20 UT. The sunspot was scanned with "fast-map" observing mode by the SP instrument. In this mode the spatial sampling along the slit is 0.32 " per pixel and across the slit is 0.29 " per pixel with integration time of 1.6 seconds. The spectral lines used for polarimetric measurement are Fe I 6301.5 and $6302.5 \AA$ line pair. The vector magnetic field for this sunspot was derived using Milne-Eddington code named MERLIN, provided under Community Spectro-polarimetry Analysis Center (CSAC) (Lites et al. 2007). It is an inversion code based on the least-squares fitting of the observed Stokes profiles using the Levenberg-Marquardt algorithm and is quick due to parallel computing. It assumes a standard Milne-Eddington atmosphere to retrieve the magnetic field vector, line-of-sight velocity, source function, Doppler broadening as well as the macro-turbulence and the stray light filling factor. For Monte-Carlo estimation of error in magnetic parameters we use another Milne-Eddington inversion code named Helix (Lagg et al. 2004). We compared the magnetic field parameters retrieved by the two codes after inverting the SOT/SP dataset used in the present study. We made difference maps of the retrieved magnetic field parameters i.e., $\delta M=M_{M E R L I N}-M_{H E L I X}$, where $M=B$ (field strength), $\gamma$ (inclination) or $\psi$ (azimuth). Within the sunspot, the two codes retrieve identical magnetic 
field parameters giving zero mean of $\delta M$ and (1- $\sigma)$ standard-deviation of 50 Gauss, 0.75 degrees and 2.5 degrees in field-strength, inclination and azimuth angle, respectively. These values are comparable to the standard errors of inversion by MERLIN, reported in figure 3. Standard errors mean that if same inversion code is repeatedly applied to a given observed (noisy) profile, it will lead to same model atmosphere within the error bars given by the standard error estimate, provided the minimum of the merit function is reached

(Bellot Rubio et al. 2000). So, the comparison suggests that the two codes are identical such that the differences in the output are of same magnitude as the difference of a particular code with itself. The reason for choosing Helix over MERLIN in our Monte-Carlo error estimation is the in-built routines for adding noise to the synthetic Stokes profiles in Helix code which helps us save time as we avoid adding noise offline. The azimuthal ambiguity of the transverse component of the magnetic field vector was resolved using acute angle method (Harvey 1969).

\subsection{The Monte-Carlo Approach for Determination of Uncertainties}

The uncertainties in the field parameters derived using ME inversion of observed Stokes profiles, purely as a result of polarimetric noise present in the observed data, are considered here. There are two ways of determining the uncertainties:

(i) Standard Errors : Basically, in the Stokes profile inversions, a merit function is defined as

$$
\chi^{2}=\frac{1}{\nu} \sum_{k=1}^{4} \sum_{i=1}^{M}\left[I_{k}^{o b s}\left(\lambda_{i}\right)-I_{k}^{s y n}\left(a, \lambda_{i}\right)\right]^{2} \frac{w_{k i}^{2}}{\sigma_{k i}^{2}}
$$

where $k=1$ to 4 represent the four Stokes parameters, $i=1$ to $M$ represent the number of wavelength samples for observed and synthetic Stokes profiles, $\nu$ represents the number of degrees of freedom (i.e., the number of observables minus the number of free parameters), and the vector $a$ consists the parameters of the model, $\sigma_{k i}$ represent the noise in the 
observations and $w_{k i}$ represents the weight given to the data points. This merit function, $\chi^{2}$, is then minimized by using non-linear least squares method to obtain $\chi_{\min }$. The standard errors in the model parameters basically depend upon the curvature of the $\chi^{2}$ function near the region of minimum in the parameter space (Press et al. 1986). More details about the estimation of the standard errors in the model parameters derived by Stokes inversion is given in Bellot Rubio et al. (2000).

(ii) Monte-Carlo Errors: In Monte-Carlo method we create several artificial realizations of the spectro-polarimetric data. This is done by generating the synthetic Stokes profiles and adding different realizations of noise to them. These artificial datasets are then inverted to obtain the magnetic parameters, leading to a collection of results. The errors are then determined by finding the spread or standard deviation in the derived parameters. It may be noted that the Monte-Carlo errors have no obvious relationship with the standard errors.

Westendorp Plaza et al. (2001) has shown that in case of SIR inversion of a single profile, the standard errors are slightly larger than the estimates from Monte-Carlo method. However, this depends on the distribution of field vector, and so the comparison of errors by the two methods may vary from pixel to pixel. Further, the Monte-Carlo error estimates depend upon the noise in the data as well as the noise in the process of inversion. Thus these are useful to establish the statistical significance of the results.

We use Monte-Carlo approach to determine the statistical spread or uncertainty in the magnetic field parameters as well as the non-potentiality parameters of active region arising due to polarimetric noise. The approach is as follows:

(1) First we take real observations of a sunspot by Hinode SOT/SP and invert it with a Milne-Eddington code to retrieve magnetic field vector.

(2) resolve the azimuthal ambiguity in the transverse field using acute angle method.

(3) consider this vector field as the "true field" $\left[B_{0}, \gamma_{0}, \psi_{0}\right]$, and the derived quantities 
$\alpha\left(B_{0}, \gamma_{0}, \psi_{0}\right)$ and $\widehat{\Psi}\left(B_{0}, \gamma_{0}, \psi_{0}\right)$ as "true non-potentiality parameters" $\left[\alpha_{0}, \widehat{\Psi}_{0}\right]$, for reference. (4) using the "true field" value at each pixel we generate synthetic Stokes profiles and add polarimetric noise of level $0.5 \%$ of continuum intensity. These profiles are then inverted again under ME approximation to derive field vector $[B, \gamma, \psi]$ and $\left[\alpha_{g}, \widehat{\Psi}\right]$. This process is repeated 100 times, each time with different realization of polarimetric noise. The uncertainty in the field strength, inclination and azimuth for each pixel and the uncertainty in the parameters $\alpha_{g}$ and $\widehat{\Psi}$ for the vector map is then estimated from the 1- $\sigma$ standard deviation in the 100 values of these parameters. This standard deviation is called the Monte-Carlo error.

This simulation gives us an idea about the spread (standard deviation) in derived field vector and the non-potentiality parameters of a real sunspot, arising due to polarimetric noise. This knowledge of standard deviation in the magnetic non-potentiality parameters $\alpha_{g}$ and $\widehat{\Psi}$ is important to determine whether the observed changes in these parameters, for example in relation to flares, are significant or not. Such studies are yet to be done and the present work will establish the level of uncertainties in the parameter $\widehat{\Psi}$ or $\alpha_{g}$ due to polarimetric noise in modern observations, such as from Hinode.

\section{3. $\quad$ Method of Adding the Noise}

We first generate synthetic Stokes profiles corresponding to the "true field" using Milne-Eddington based Stokes profile synthesis and inversion code named Helix (Lagg et al. 2004). To these synthetic profiles we add normally distributed random noise with the 3- $\sigma$ level of $0.5 \%$ of continuum intensity $\left(I_{c}\right)$. The noise, $N(\lambda)$, is added to the synthetic Stokes profiles, $S_{\text {syn }}(\lambda)$, as follows:

(1) First a pseudo random-number sequence is generated which is normally distributed with 
a zero mean and a $3-\sigma$ standard deviation of given level, say L. In our case $\mathrm{L}=0.5 \%$ of $I_{c}$.

$$
N(\lambda)=\text { Random_Number }(\text { Seed }, L)
$$

(2) This sequence is then added to the synthetic Stokes profiles to yield noisy Stokes profile, $S_{\text {noi }}(\lambda)$.

$$
S_{\text {noi }}(\lambda)=S_{\text {syn }}(\lambda)+N(\lambda)
$$

The noise level is estimated from the observed signals in the continuum of the Stokes spectra. A continuum window between $6302.83 \AA$ and 6303.28 Ais selected for monitoring the noise in Stokes signal. The left panel of the figure 1 shows the histogram of noise in the observed Stokes profiles for a large number of pixels (1024 pixels) for a typical SOT/SP scan in "fast-map" observing mode. A Gaussian fit to this distribution yields a 3- $\sigma$ value of $0.5 \%$ of $I_{c}$. This is the noise level that we used in our simulations. The histogram in the right panel of figure 1 shows the distribution of artificial noise that we add to the synthetic profiles. These profiles are then inverted with the Helix code. This process is repeated 100 times and so for each pixel we have 100 values distributed around a mean value.

While modern spectro-polarimetric observations have typically noise levels of the order of $0.5 \%$ of $I_{c}$, archived observations from the ground-based instruments, which might be used for synoptic studies, may have higher levels of noise. Therefore, we also carried out an exercise to check the variation in the magnetic as well as non-potentiality parameters, $\widehat{\Psi}$ and $\alpha_{g}$, with increasing polarimetric noise levels. We added four different levels of noise, i.e., $0.01,0.1,0.5$ and $1 \%$ of continuum intensity.

\subsection{Method of computing $\alpha_{g}$ and $\widehat{\Psi}$}

The computation of the non-potentiality parameter $\alpha_{g}$ and $\widehat{\Psi}$ is done as proposed by Tiwari et al. (2009a, 2009b). The expression for computation of these parameters is given 
in equations (1) and (2) in the introduction section. Only those pixels which have field values above a certain noise level are analyzed. This filtering is done in the following way: we select a quiet region on the Sun and evaluate 1- $\sigma$ deviation in the three vector field components $B_{x}, B_{y}$ and $B_{z}$ separately. The box selected for this estimation is shown in top-left panel of figure 2, For transverse vector fields, we take 1- $\sigma$ noise level as the resultant deviations obtained in $B_{x}$ and $B_{y}$. Only those pixels where transverse and line-of-sight (LOS) field both are together greater than twice the above mentioned noise level of $1-\sigma$ are analyzed. The 1- $\sigma$ value for longitudinal and transverse field inside the box is 380 and 258 Gauss, respectively.

\section{Results}

\section{1. $\quad$ Effect of noise on magnetic field parameters}

The figure 2 shows the effect of noise on magnetic field parameters for the NOAA 10933 sunspot. The top row shows the initial magnetic field parameters $\left[B_{0}, \gamma_{0}, \psi_{0}\right]$ derived by ME inversion of Stokes profiles obtained by Hinode SOT/SP scan during 07:20 UT on Jan 5, 2009. The middle row shows the map of Monte-Carlo error, i.e., the 1- $\sigma$ standard deviation in the magnetic field parameters, derived using the Monte-Carlo method. The

bottom row shows the map of standard error in the field parameters from the least-squares fit of the Stokes profiles. The maximum error (taking into account both the standard error and Monte-Carlo error estimate) inside the sunspot is less than 50 Gauss for the field strength. While for inclination and azimuth it is less than few degrees. The azimuth errors are typically largest in the umbral and plage region where the field is almost vertical and azimuth is not well defined.

In figure 2, we isolate the umbral and penumbral regions by using continuum intensity 
thresholds. We compare the standard errors and the Monte-Carlo errors in these two regions in the figure 3. The number of pixels in the umbral and penumbral regions plotted in figure 3 are 1953 and 13615, respectively. It may be seen that :

(i) in the umbral and penumbral regions the Monte-Carlo errors are typically less than 5 Gauss and 8 Gauss for field strength, less than 1 and 0.5 degrees for field inclination, and less than 5 and 2 degrees for field azimuth, respectively.

(ii) the standard error is larger than Monte-Carlo error estimates in both the umbral and penumbral regions for field strength as was found by Westendorp Plaza et al. (2001) in case of the inversion of a single profile. While for field azimuth and inclination it has an opposite relation, except for middle panel of bottom row.

The standard error depends upon the sensitivity of the $\chi^{2}$ to the changes in a particular parameter. It can so happen that the Stokes profiles might not have significant sensitivity to the changes in that parameter. This results in a large standard error. On the other hand, the Monte-Carlo method examines the changes in the derived parameters resulting from the changes in the Stokes profiles arising due to random fluctuations in the profile as a result of different realizations of noise being added. Since both errors depend upon different origins of the profile fluctuations, they need not produce the same results.

In general, considering all the panels in figure 3, it may be concluded that the standard errors are larger than the Monte-Carlo errors. Here, one should bear in mind that standard errors shown in figure 3, are for inversion of real Stokes profiles which possess asymmetry, apart from the polarimetric noise. While, Monte-Carlo error correspond to repeated inversion of purely synthetic, and therefore symmetric (or antisymmetric for Stokes-V) profiles with polarimetric noise. Therefore, the main source of difference between the two type of errors could be attributed to the presence of Stokes asymmetry in the real stokes 
profiles leading to large value of standard error. Ideally, one would like to perform a Monte-Carlo error estimate by obtaining several simultaneous observational datasets of a real sunspot where the Stokes profiles would have inherent Stokes asymmetry as well as different realization of polarimetric noise. However, in the absence of such a possibility we used present method to study the effect of polarimetric noise alone, neglecting Stokes asymmetry. The results of the present study are therefore only the lower limits of the errors that can occur practically in real observations as well as in the non-potentiality parameters $\widehat{\Psi}$ and $\alpha_{g}$.

\subsection{Effect of noise on Azimuthal Ambiguity Resolution}

It is well known that the Zeeman effect diagnostics cannot detect the direction of the transverse field component and so a 180 degree ambiguity remains in the determination of the field azimuth. Various methods, however, have been developed by the researchers to resolve this ambiguity using different arguments (Leka et al. 2009). One of the most common and widely applicable method is the so-called acute angle method. In this method the angle between the observed and potential transverse field, i.e., $\theta=\operatorname{acos}\left(B_{o}^{o b s} \cdot B_{t}^{p o t}\right) /\left(\left|B_{o}^{o b s} \| B_{t}^{p o t}\right|\right)$, is computed and the solution for which the value of $\theta$ is an acute angle is considered as the correct solution. However, in the presence of polarimetric noise in the observations, this method also can fail specially in situations where the observed field is highly sheared. Such highly sheared regions are found near polarity inversion line (PIL) of the active regions.

Fortunately, in a normal round sunspot, like the one used in our simulations, there are no high-shear regions and therefore the azimuthal ambiguity is easily solved with acute angle method. Nevertheless, we need to check the effect of polarimetric noise on the azimuth

ambiguity resolution in our simulations before we examine the uncertainty in the $\widehat{\Psi}$ and $\alpha_{g}$ 
parameter of the sunspot in 100 realizations. After resolving the azimuthal ambiguity using acute angle method for the 100 realizations of the sunspot vector maps, we made a map of 1- $\sigma$ standard deviation for azimuth angle, as shown in middle-right panel of figure 2. If the ambiguity is not resolved properly in these 100 realizations, there will be fluctuations of the order of 180 degrees, leading to a large value of standard deviation in azimuth value for a given pixel. However, in the right panel of the middle row in figure 2 we see that:

(i) the resolution of the azimuth angle is quite stable for the most part within the sunspot, specially in the penumbral region. Outside the sunspot, in the quiet and facular areas, the errors are large. This is mainly due to poor SNR in these areas as a result of weaker and/or vertical fields.

(ii) As we go towards the umbra the values of standard deviation are large. We examined the values of the field azimuth in these regions and found that values do not vary to the extent of 180 degrees and therefore the large errors in umbral region is not due to ambiguity solver but due to poor SNR in Stokes-Q and U observations. Only in the very central part of the umbra, where the field inclination is close to 90 degrees, the azimuth loses its meaning and so we see a large spread.

Further, we also checked the effect of ambiguity solver with increasing noise i.e., four different levels of noise of $0.01,0.1,0.5$ and $1 \%$ of continuum intensity. Here also the azimuth ambiguity resolution is stable for most part of the sunspot.

\section{3. $\quad$ Effect of noise on $\widehat{\Psi}$ and $\alpha_{g}$}

The figure 4 shows histogram of $\widehat{\Psi}$ and $\alpha_{g}$ corresponding to 100 Monte-Carlo realizations of vector field. It can be noticed that:

1. The $\widehat{\Psi}$ is not affected much by noise: The distribution of $\widehat{\Psi}$ corresponding to 100 realizations show less scatter $(\sim 1 \%)$ with $\widehat{\Psi}=-1.68 \pm 0.014^{\circ}$. 
2. The values of $\alpha_{g}$ are more affected: The distribution shows large scatter $(\sim 10 \%)$ in values with $\alpha_{g}=-3.5 \pm 0.37\left(\times 10^{-9} m^{-1}\right)$.

These results show that the $\widehat{\Psi}$ may be more reliable as compared to $\alpha_{g}$ for typical polarimetric noise present in modern spectro-polarimetric observations as it is a lower dispersion parameter.

We also check the effect of increasing noise in the polarimetric measurements on the magnetic as well as non-potentiality parameters of the same sunspot. In order to compare the effect of increasing noise on the field parameters in the sunspot we isolate the umbral and penumbral region, as was done in figure 2, and do a scatter plot between the input and output field parameters, for different levels of noise. The scatter plot for umbral and penumbral region is shown in figure 5 and figure 6 respectively. It can be seen that, in the umbra, where the field is mostly vertical, the azimuth determination is more affected with increasing noise than the field strength and inclination. Specially, for $0.5 \%$ and $1 \%$ noise levels the spread is large in the azimuth and inclination values. In comparison, the scatter plot for the penumbral region, where the field is not so vertical, shows that the azimuth is determined with less spread even for $0.5 \%$ and $1 \%$ noise levels, respectively.

Table 1 lists the non-potentiality parameters, derived from the ME inverted magnetic field vector, after adding noise of different levels in the synthetic Stokes profiles. In Table 1, the spatial fluctuations of the shear angle $\Psi$ as well as local $\alpha$ in the map, are represented as $\sigma_{\Psi}$ and $\sigma_{\alpha}$. Starting with no noise and then adding random noise of $0.01,0.1,0.5$ and $1 \%$ of continuum intensity in the Stokes profiles we get the following results: (i) The sign of the twist in the sunspot magnetic field, as inferred by both $\alpha_{g}$ and $\widehat{\Psi}$, is negative and is not affected even when the SNR of Stokes profiles is poor, (ii) the absolute value of $\widehat{\Psi}$ tends to decrease systematically with increasing noise in the Stokes profiles, and (iii) the fluctuation of $2.5 \%$ in the $\widehat{\Psi}=-1.65 \pm 0.04^{\circ}$ is less than the fluctuation of about $5 \%$ in 
$\alpha_{g}=3.15 \pm 0.17\left(\times 10^{-9} m^{-1}\right)$.

\section{Discussion}

Developing quantitative measures of magnetic non-potentiality in solar active regions is very important for flare research. The line-of-sight magnetic field alone is insufficient for this purpose, therefore people started measuring vector magnetic fields. The stresses in the magnetic field were quantified in terms of the magnetic shear angle, by comparing the observed field with the potential-field (or current-free fields). The distribution of shear angle over active region showed that the filament bearing PIL regions are characterized by high-shear and are potential sites for flares. Shear, as a local measure of non-potentiality can be easily extended as a whole active region measure, like SASSA $\widehat{\Psi}$ (Tiwari et al. 2009b).

Other physical measures of whole active region non-potentiality are the virial estimate of free-energy and $\alpha_{g}$. Both of these measures require the photospheric magnetic field to be force-free. However, force-free condition may not be justified as the plasma $\beta$ in the photosphere is not much smaller than unity and so the non-magnetic forces are not negligible. The effect of the polarimetric noise on the virial free-energy estimate was determined by Klimchuck et al. (1992) and Tiwari et al. (2009a). However, the results of these studies are valid for analytic force-free field solution of (Low 1982). While, in real

sunspots, the effect of non-force-freeness of photospheric field on virial energy and $\alpha_{g}$ may be larger than the effect of polarimetric errors quantified by Klimchuck et al. (1992) and Tiwari et al. (2009a). The whole active region non-potentiality characterized by SASSA $\widehat{\Psi}$ is, however, free of force-free assumption and in the present work we studied the effect of polarimetric noise on this parameter. 
We simulated polarimetric profiles with noise level of $0.5 \%$ of $I_{c}$, which is typical of Hinode SOT/SP observations as shown in figure 1. It must be noted that the synthesis of these profiles is done under the assumption of ME model atmosphere and so the present results pertain to symmetric Stokes profiles only. The asymmetry of the Stokes profiles would lead to a systematic rather than random effect which needs to be quantified in a separate study. In hundred realizations of such noisy symmetric Stokes profiles we found that the parameter $\widehat{\Psi}$ is statistically more stable than $\alpha_{g}$. The reason for this stability of $\widehat{\Psi}$ as compared to $\alpha_{g}$ can be understood as follows. In Eqn. (2) $\widehat{\Psi}$ is derived as a simple summation of angles. So, the random errors would cancel each other in summation. While, in Eqn. (1) we note that $\alpha_{g}$ depends upon three components $B_{x}, B_{y}$ and $B_{z}$ and is not a simple summation and therefore the random errors in the three parameters would not vanish statistically. Thus, $\alpha_{g}$ would be more vulnerable to the noise as compared to $\widehat{\Psi}$.

Further, in the presence of different noise levels i.e., $0.01,0.1,0.5$ and $1 \%$ of $I_{c}$, it is found that the standard deviation in $\widehat{\Psi}$ is less vulnerable to increasing noise than $\alpha_{g}$. The absolute value of $\widehat{\Psi}$ tends to reduce systematically with increasing noise.

Further, one should keep in mind that the accuracy of derived magnetic field parameters also depends upon the number of free parameters (parameters of the model atmosphere) used in the fitting procedure. The present results pertain to a single-component model atmosphere with stray-light component. The results would be different when one employs more number of free parameters, like two-component model atmosphere (Leka 2001; Lites et al. 2002) or depth-dependent (stratified) model atmosphere used in SIR inversions. In general the uncertainty in the best-fit model parameters will deteriorate with increasing number of model (or free) parameters. 


\section{Conclusion}

We generated an ensemble of artificial spectro-polarimetric dataset for a given sunspot. This ensemble of data is then inverted to give an ensemble of vector maps. These maps are then used to estimate of uncertainties in the field parameters as well as in the non-potentiality parameters, $\alpha_{g}$ and $\widehat{\Psi}$, of the sunspot. The standard errors of ME fitting are given by the inversion codes according to Press et al. (1986) and Bellot Rubio et al. (2000). The Monte-Carlo errors give us an independent method to cross-check the standard error estimates. In figure 3, we show that the Monte-Carlo errors for field strength, inclination and azimuth in the sunspot is determined within $\pm 8 \mathrm{G}, \pm 1$ degree and \pm 5 degree, respectively. A comparison shows that the Monte-Carlo errors in field strength are typically smaller than the standard errors, while it is opposite for the field azimuth and inclination, except for the field inclination in penumbra where again the Monte-Carlo errors are less than standard errors. In general, the standard errors are more conservative estimates because they include the effects of polarimetric noise as well as those of Stokes asymmetry, while Monte-Carlo errors account only for the polarimetric noise.

The effect of polarimetric noise on the parameters characterizing the non-potentiality of a sunspot magnetic field suggests that $\widehat{\Psi}$ is more robust than $\alpha_{g}$ (as shown by the histogram in figure 4). Further, $\widehat{\Psi}$ appears to be a stable parameter with increasing noise in the polarimetric data (Table 1).

Thus, $\widehat{\Psi}$ will be useful for studying the evolution of non-potentiality of the active region magnetic fields, which in turn, can help in the prediction of flare occurrence. The

parameter $\widehat{\Psi}$ in a large number of active regions as well as the evolution of $\widehat{\Psi}$ in a flaring regions will be evaluated in a future work.

We thank the referee for detailed comments and suggestions. We thank Dr. Bruce 
Lites for helping in the estimation of the standard errors. We also would like to thank Dr. Andreas Lagg for providing his HeliX code used in this study. MERLIN inversions of Hinode SOT/SP were conducted at NCAR under the framework of the Community Spectro-polarimtetric Analysis Center (CSAC). Hinode is a Japanese mission developed and launched by ISAS/JAXA, with NAOJ as domestic partner and NASA and STFC (UK) as international partners. It is operated by these agencies in co-operation with ESA and NSC (Norway). We thank the IFCPAR (Indo-French Centre for Promotion of Advanced Research) for the financial support under Project 3704-1. 


\section{REFERENCES}

Abramenko, V. I., Wang, T., \& Yurchishin, V. B. 1996, Sol. Phys., 168, 75

Bao, S., \& Zhang, H. 1998, ApJ, 496, L43+

Bellot Rubio, L. R., Ruiz Cobo, B. \& Collados, M. 2000, ApJ, 535, 475

Chatterjee, P., Choudhuri, A. R., \& Petrovay, K. 2006, A\&A, 449, 781

Démoulin, P., \& Pariat, E. 2009, Advances in Space Research, 43, 1013

Falconer, D. A., Moore, R. L., \& Gary, G. A. 2008, ApJ, 689, 1433

Gosain, S., Venkatakrishnan, P., \& Tiwari, S. K. 2009, ApJ, 706, L240

Gosain, S., Venkatakrishnan, P., \& Venugopalan, K. 2004, Experimental Astronomy, 18, 31

Gosain, S., Venkatakrishnan, P., \& Venugopalan, K. 2006, Journal of Astrophysics and Astronomy, 27, 285

Hagino, M., \& Sakurai, T. 2004, PASJ, 56, 831

-. 2005, PASJ, 57, 481

Harvey, J. W. 1969, PhD thesis, UNIVERSITY OF COLORADO AT BOULDER.

Ichimoto, K., et al. 2008, Sol. Phys., 249, 233

Klimchuk, J. A., Canfield, R. C., \& Rhoads, J. E. 1992, ApJ, 385, 327

Kosugi, T., et al. 2007, Sol. Phys., 243, 3

Lagg, A., Woch, J., Krupp, N., \& Solanki, S. K. 2004, A\&A, 414, 1109

Leka, K. D. 2001, Advanced Solar Polarimetry - Theory, Observation, and Instrumentation, 236, 571 
Lites, B. W., Socas-Navarro, H., Skumanich, A., \& Shimizu, T. 2002, ApJ, 575, 1131

Lites, B. W., et al. 2007, in Astronomical Society of the Pacific Conference Series, Vol. 369, New Solar Physics with Solar-B Mission, ed. K. Shibata, S. Nagata, \& T. Sakurai, $55^{-+}$

Low, B. C. 1982, Sol. Phys., 77, 43

Mickey, D. L., Canfield, R. C., Labonte, B. J., Leka, K. D., Waterson, M. F., \& Weber, H. M. 1996, Sol. Phys., 168, 229

Nandy, D. 2006, Journal of Geophysical Research (Space Physics), 111, 12

Pevtsov, A. A., Canfield, R. C., \& Metcalf, T. R. 1994, ApJ, 425, L117

—. 1995, ApJ, 440, L109

Press, W. H., Flannery, B. P., \& Teukolsky, S. A. 1986, Numerical recipes. The art of scientific computing, ed. Press, W. H., Flannery, B. P., \& Teukolsky, S. A.

Ruiz Cobo, B., \& del Toro Iniesta, J. C. 1992, ApJ, 398, 375

Scherrer, P. H., \& SDO/HMI Team. 2002, in Bulletin of the American Astronomical Society, Vol. 34, Bulletin of the American Astronomical Society, 735-+

Su, J. T., Sakurai, T., Suematsu, Y., Hagino, M., \& Liu, Y. 2009, ApJ, 697, L103

Tiwari, S. K., Venkatakrishnan, P., Gosain, S., \& Joshi, J. 2009a, ApJ, 700, 199

Tiwari, S. K., Venkatakrishnan, P., \& Sankarasubramanian, K. 2009b, ApJ, 702, L133

Tsuneta, S., et al. 2008, Sol. Phys., 249, 167

Venkatakrishnan, P., \& Tiwari, S. K. 2009, ApJ, 706, L114 
Westendorp Plaza, C., del Toro Iniesta, J. C., Ruiz Cobo, B., Martinez Pillet, V., Lites, B. W., \& Skumanich, A. 2001, ApJ, 547, 1130 

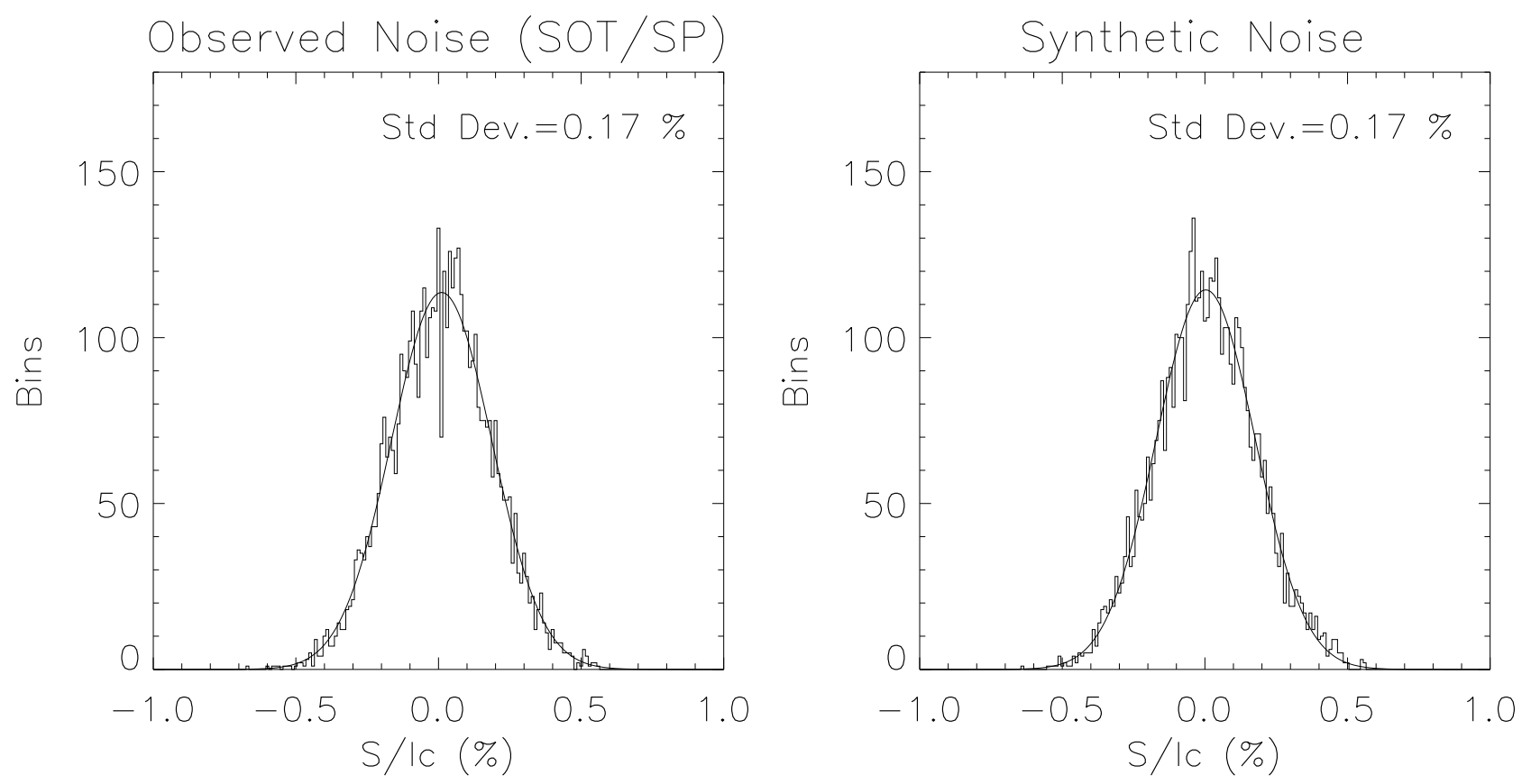

Fig. 1. - The left panel shows the histogram of the noise in the Stokes profiles $\left(S / I_{c}\right)$ for typical SOT/SP "fast-mode" scan. The right panel shows the histogram of normally distributed psuedo-noise added in the synthetic profiles for Monte-Carlo simulations. The solid line shows the fitted Gaussian with $1-\sigma$ noise level is about $0.17 \%$ of $I_{c}$. 

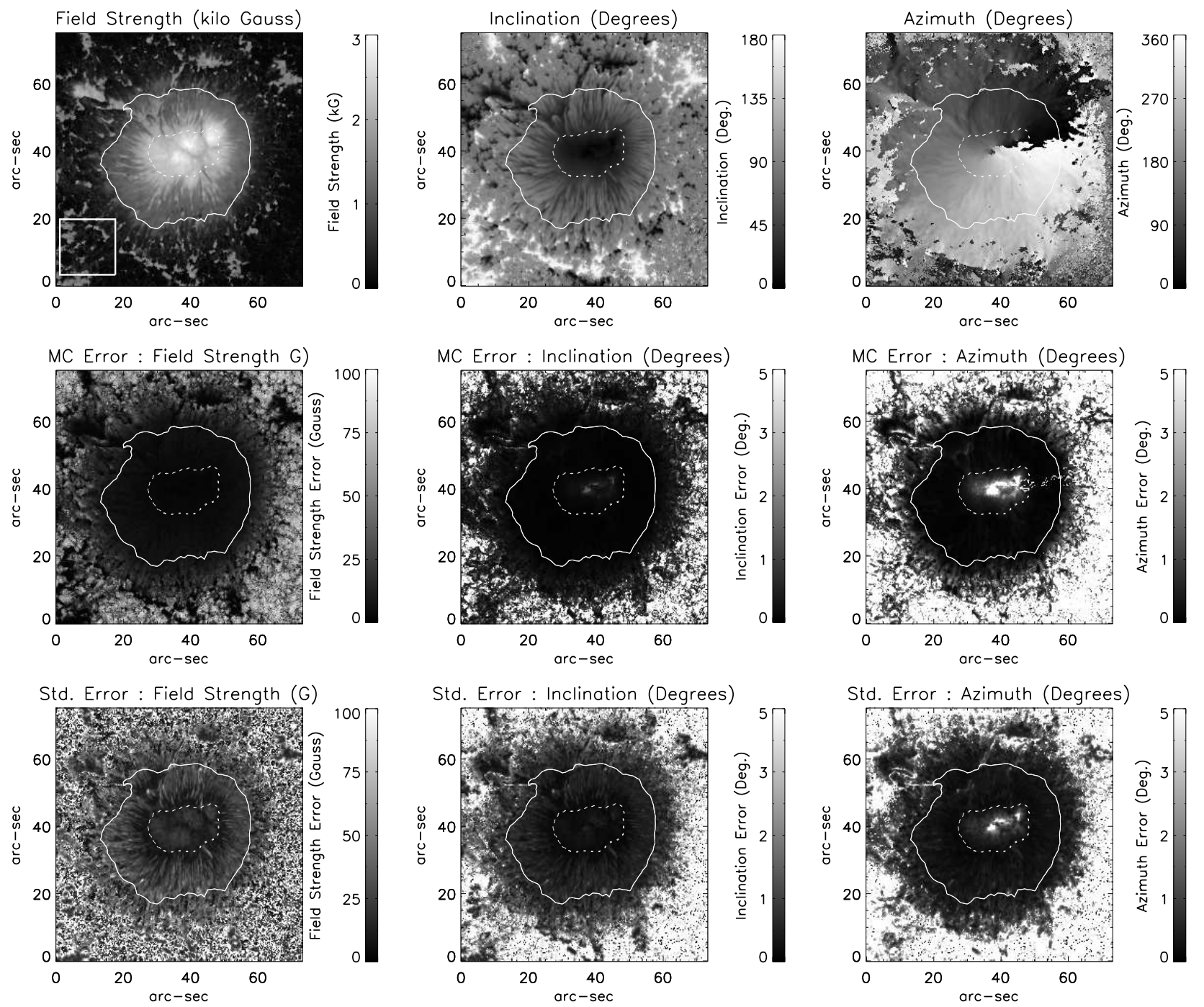

Fig. 2.- The top row shows the maps of magnetic field strength, inclination and azimuth for the sunspot in NOAA 10933 derived using Milne-Eddington inversion. The solid and dashed line contours mark the penumbral and umbral regions, respectively. The white box in top left panel marks the box used for estimation of noise levels in magnetic field components. The middle row shows the error maps (1- $\sigma$ standard deviation) for field strength, inclination and azimuth derived using Monte-Carlo method. The bottom row shows the maps of the standard error in Milne-Eddington fitting for the field strength, inclination and azimuth. 

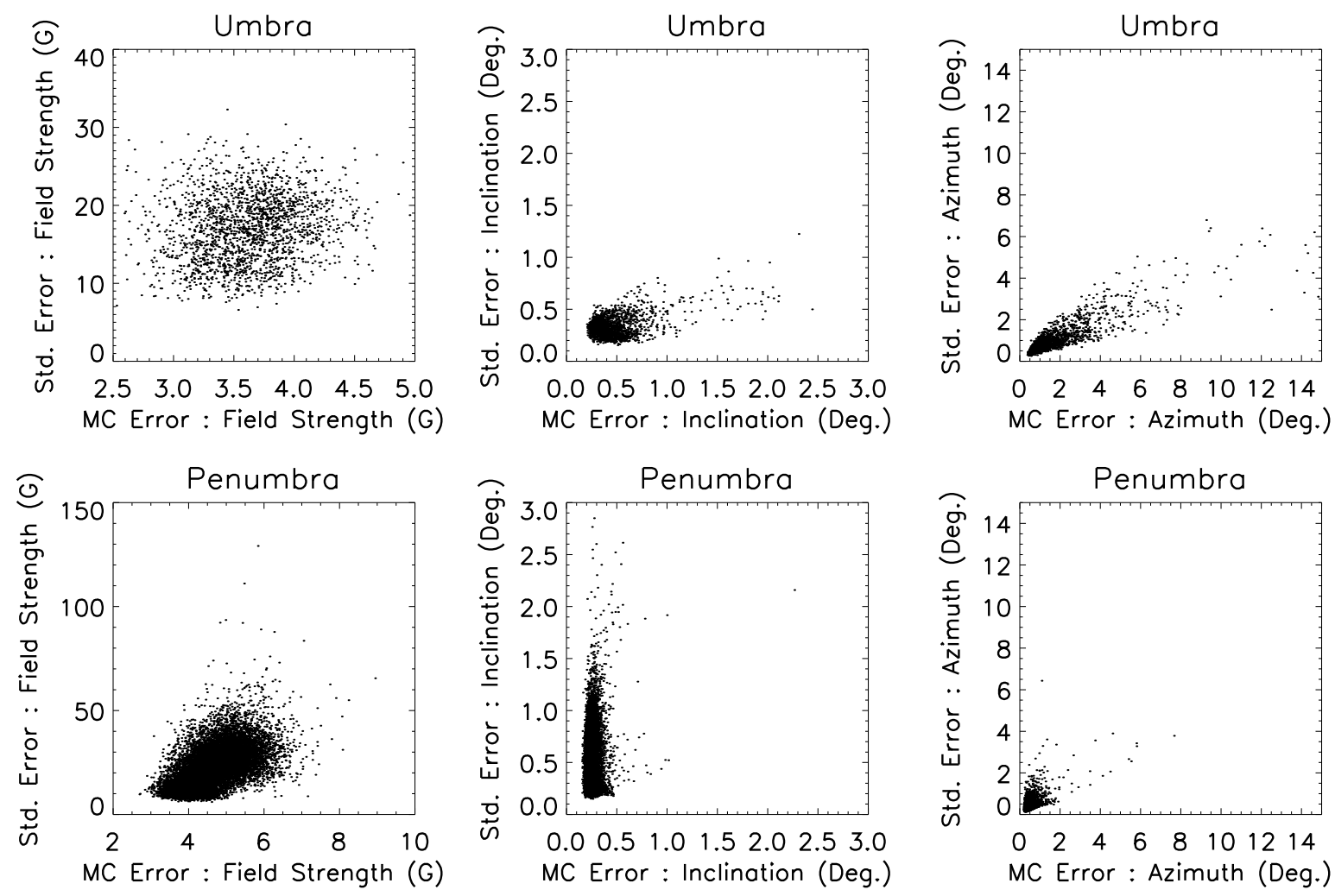

Fig. 3.- Top panel: The scatter plot between the Monte-Carlo errors and standard errors of fitting are shown for field strength, inclination and azimuth for all pixels in umbral region. Bottom panel: The scatter plots for pixels inside penumbral region. 

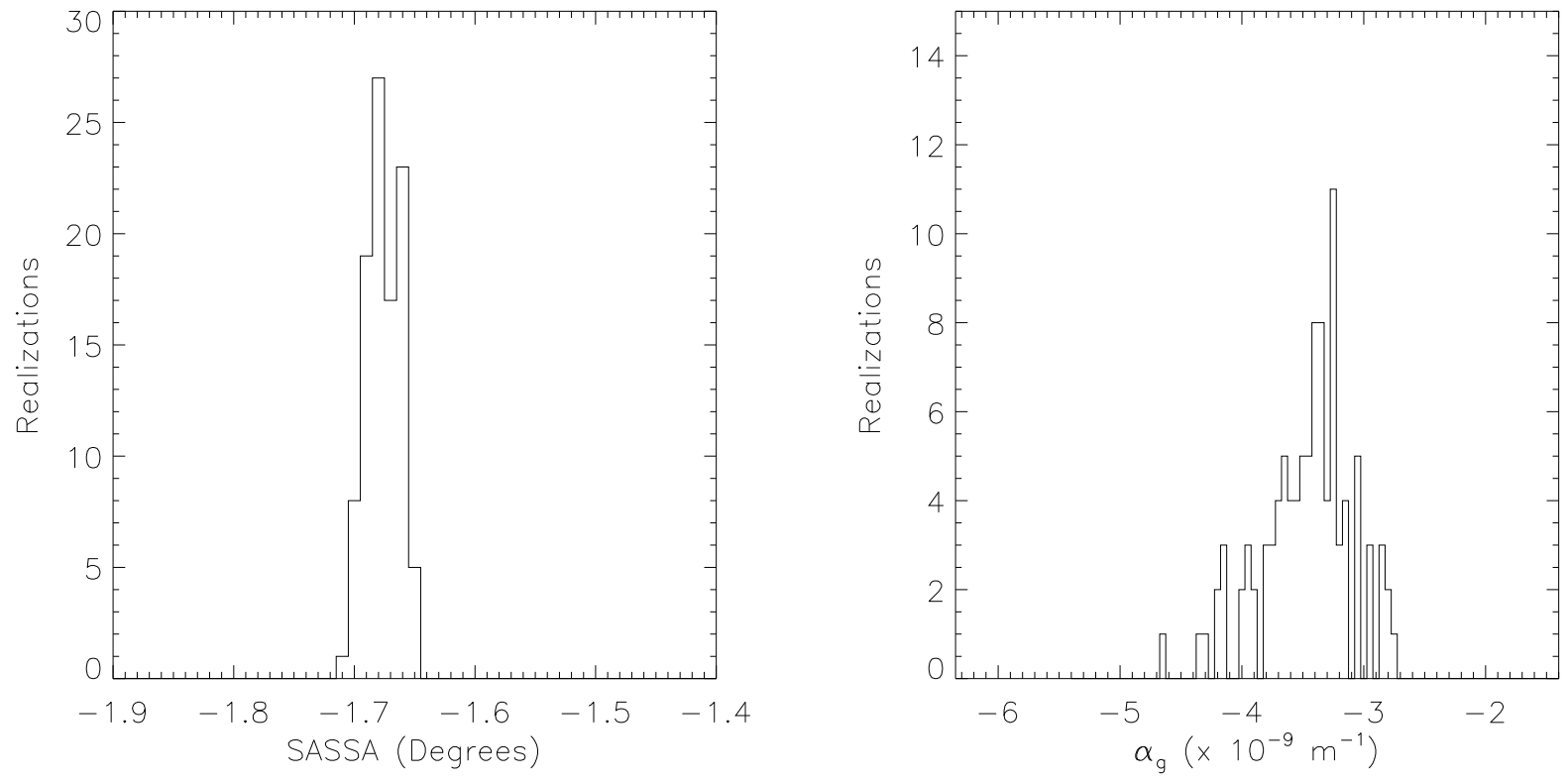

Fig. 4.- The histograms show distribution of $\widehat{\Psi}$ and $\alpha_{g}$ corresponding to NOAA 10933. The vector maps were derived hundred times, and each time different realization of normally distributed noise $\left(3-\sigma=0.5 \%\right.$ of $\left.I_{c}\right)$ is added to the synthetic profiles. 

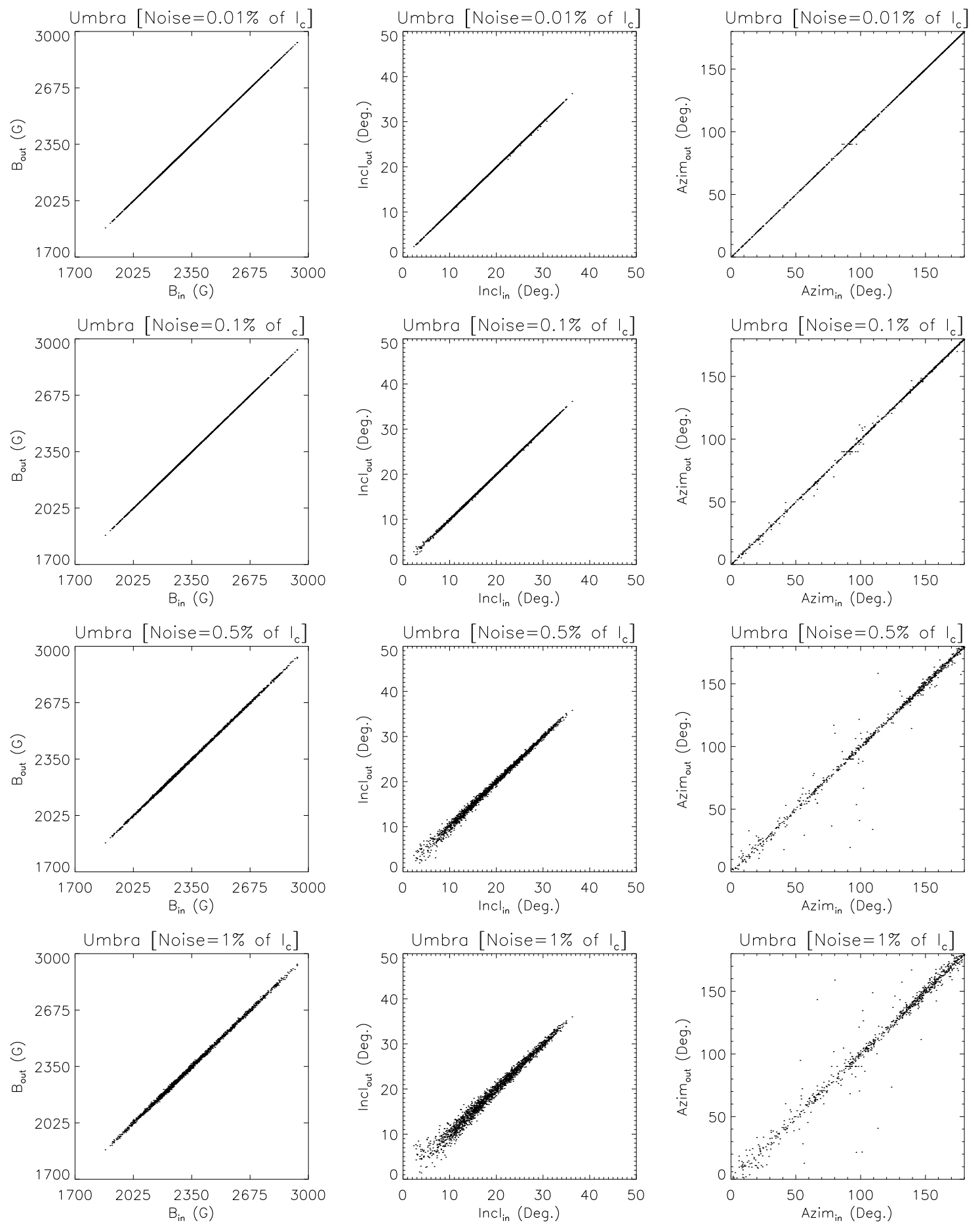

Fig. 5.- The scatter plots between the input and derived magnetic field parameters after different level of noise is added to Stokes profiles. The top of each panel shows the 3- $\sigma$ level of normally distributed noise that is added to the synthetic profiles. The $\mathrm{x}$-axis shows the input magnetic field parameter used in the profile synthesis and the y-axis shows the output value of the parameter derived from the noise added synthetic profile. 

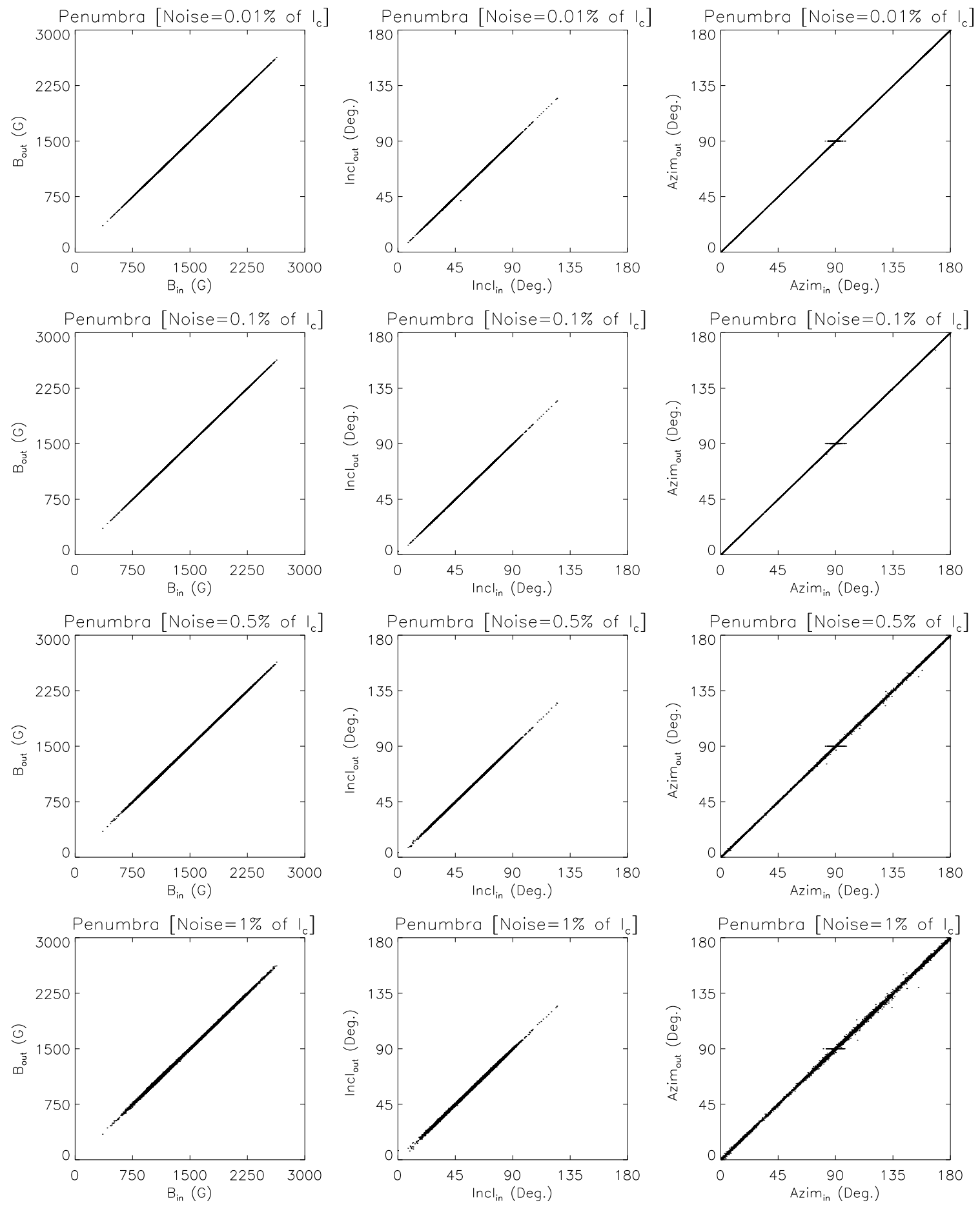

Fig. 6.- Same as figure 7, but for penumbral region. 
Table 1: Effect of increasing polarimetric noise in the estimation of $\widehat{\Psi}$ and $\alpha_{g}$

\begin{tabular}{lrrcr}
\hline Noise $\left(\%\right.$ of $\left.I_{c}\right)$ & $\widehat{\Psi}\left(^{\circ}\right)$ & $\sigma_{\Psi}\left(^{\circ}\right)$ & $\alpha_{g}\left(m^{-1}\right)$ & $\sigma_{\alpha}\left(m^{-1}\right)$ \\
\hline 0 & -1.692 & 15.724 & $-3.334 \times 10^{-9}$ & $3.572 \times 10^{-7}$ \\
0.01 & -1.688 & 15.726 & $-3.360 \times 10^{-9}$ & $3.573 \times 10^{-7}$ \\
0.1 & -1.685 & 15.702 & $-3.221 \times 10^{-9}$ & $3.574 \times 10^{-7}$ \\
0.5 & -1.648 & 15.636 & $-2.976 \times 10^{-9}$ & $3.562 \times 10^{-7}$ \\
1 & -1.604 & 15.364 & $-3.026 \times 10^{-9}$ & $3.539 \times 10^{-7}$ \\
\hline
\end{tabular}

